\title{
A novel way to estimate the epidemic risk index for assessing the government policies during the epidemic of respiratory transmitted person-to-person disease
}

\author{
Dimitrije D. Čvokić
}

Received: date / Accepted: date

\begin{abstract}
The epidemic risk index is formulated in a novel way, extending the INFORM risk formula. The development is based on the well known gravity model. Modularity of the proposed epidemic risk index allows ad hoc modifications according to the current knowledge of biological pathogen which is a cause of epidemic and available data about geographical areas of interest. Concerning the SARS-CoV-2 virus pandemic in 2020, the model is presented so that it can be usable, hopefully, in the current situation, although, in general, it should be applicable to any respiratory transmitted person-to-person disease.
\end{abstract}

Keywords epidemic management · epidemic risk index · gravity model

Mathematics Subject Classification (2010) 92C60 -97M60 - 62-07

\section{Introduction}

An epidemic is a rapid spread of disease in a given population within a relatively short period of time. Preparations for an epidemic include developing and having a surveillance system and working on the system for quick dispatch of emergency workers. Epidemic management is the process of anticipating, preparing for, preventing, detecting, responding, and controlling epidemics in order to minimize its health, social and economic effects. These actions are guided, monitored, and evaluated by management committees [1]. An interested reader is referred to papers $[2,3]$ and World Health Organization (WHO) handbook [4] in order to get more informed about this topic.

D.D. Čvokić

University of Banja Luka

Tel.: +38751319142

E-mail: dimitrije.cvokic@pmf.unibl.org 
For a management committee, the epidemic risk index (ERI) is a mathematical tool that supports strategic decision making and prioritization of capacity building activities for national epidemic prevention, preparedness, and response [6]. The ERI evaluates national and sub-national vulnerabilities and risks to epidemic prone diseases through a data-guided mathematical model and also provides a tool to test epidemic prevention and preparedness strategies. The evaluation of ERI is scalable to a wide range of geographic are types - from countries to municipalities. Thus, the ERI can be used to strengthen or weaken the government response taken during the epidemic or disease outbreak. Too strong policy can have a devastating effect on the economy and health of people after the disease is suppressed []. On the other hand, too weak response, obviously, can expose a large quantity of people to a possibly highly lethal and contagious pathogen.

The literature is rather scarce when it comes to the ERI. Much more work has been done when it comes to the epidemic development models. In 2017 Zhu et al. [5] analyzed and proposed a reaction-diffusion-advection SIR model for the transmission of the infectious disease, for which they introduced and used a risk index for free boundary problem. Later, Doherty et al. [6] presented a JRC technical report about support collaborative risk assessment for health threats. They have proposed a formula to estimate the risk during the hazard event. A decision-support framework to optimize border control for global outbreak mitigation was proposed by Zlojutro et al. [7]. Haoxuan Sun et al. [8] have investigated a varying coefficient Susceptible-Infected-Removal model (vSIR), in which they track the epidemic of SARS-CoV-2 (COVID-19) in 30 provinces in China and 15 cities in Hubei province, the epicenter of outbreak.

In this report, a novel proposal for ERI is given, slightly focusing on the SARS-CoV-2 pandemic in 2020. Nevertheless, this ERI can, in general, be applied for any other disease outbreak on a larger or smaller scale. The rest of paper is organized as follows. In Sect. 2 the background of parameters needed to compute ERI is presented and discussed, strongly based on [6]. Afterwards, in Sect. 3, the newly proposed formula for ERI is provided. Sect. 4 presents an empirical study. Finally, in Sect. 5 some conclusions and directions of further research are given.

\section{Parameters}

In general, using the INFORM methodology [6], the risk is evaluated using the following expression:

$$
\begin{aligned}
\text { Risk }= & \text { Hazard } \times \text { Exposure } \times \\
& \text { Vulnerability } \times \text { Lack of Coping Capacities. }
\end{aligned}
$$

The older, and more widely used evaluation, excludes the lack of coping capacities, but it is obvious, especially during the occurrence of SARS-CoV-2 pandemic in 2020 [9], that the coping capacities have a great role in how a 
country or municipality can deal with an outbreak. Countries that have reacted on time, that have more ventilators and hospital beds per 1000 citizens (e.g., Japan, South Korea, Russia, Germany, Austria, China) have distinctively lower death rate $[10-12, ?]$. Furthermore, some of these countries, like Russia, China, and Cuba, are exactly the ones which are sending medical help across the world during the SARS-CoV-2 pandemic in 2020 [13-15].

Closely inspecting the Eq. (1), we can realize that it resembles the gravity law. It is well known that gravity model has also been applied to socioeconomic bilateral flow data such as migration, traffic, remittances and foreign direct investment. For more information the reader is referred to [16].

In this case, Coping Capacities has a role similar to the one that distance has in the well-known gravity law, i.e., it suppresses the effect of pathogen entity which has a power to jeopardize the vulnerable part of population. Therefore, it is natural to propose a more general formulation:

$$
E R I=\varphi \times \frac{\text { Hazard }^{\beta_{H}} \times \text { Exposure }^{\beta_{E}} \times \text { Vulnerability }^{\beta_{V}}}{\text { Coping Capacities }}{ }^{\beta_{C C}} \times \varepsilon
$$

where $\phi$ is a "gravitational constant" (a fitting coefficient), and $\beta_{H}, \beta_{E}, \beta_{V}, \beta_{C C}$ are power fitting coefficients, and $\varepsilon$ is a an error term with expectation equal to $1(E(\varepsilon)=1)$. It is assumed that all terms Hazard, Exposure and Vulnerability are non-negative and Coping Capacity is strictly greater than zero. The following arguments provide a suitable justification:

- if there exist no pathogenic entity then Hazard is 0 (otherwise it is greater than 0$)$;

- the pathogenic entity can exist and be very lethal, but if nobody is exposed to it the Exposure should take value 0 (otherwise it is greater than 0);

- the pathogenic entity can exist and population can be exposed to it in some way, but if only invulnerable part of population is exposed the Vulnerability can take value 0 (otherwise it is greater than 0 );

- if there are no epidemic committees, no epidemic management, and no hospitals, then it does not make sense to compute ERI and the domain of Coping Capacity is the set of non-negative real numbers.

Remark 1 In Eq. (2) Coping Capacities is used instead of Lack of Coping Capacities, as it looks like a more straightforward and direct way to express the idea behind ERI. The later term is used in [6].

Furthermore, the ERI can be addressed as a 4-parameter function ERI : $[0,1]^{3} \times(0,1] \rightarrow[0,1]$. The value 0 means that there exists no risk at all (an ideal, but unrealistic situation), and value 1 corresponds to a fully closed area in which the disease outbreak is at its peak, when no countermeasures are taken by a government or city authorities (also, sociologically unrealistic situation).

Definition $1 \mathrm{n}$ epidemiology a hotspot is a geographical area in which exists a high prevalence of particular disease or the population behavior puts people at risk for acquiring an infection. 
The set of all geographical locations of interest $i$ will be denoted as $I$.

\subsection{Hazard}

Because the focus of this paper is an epidemic, a hazard is a pathogenic biological entity. In the case of currently active SARS-CoV-2 pandemic in 2020, it is a virus from the corona family []. Therefore, the Hazard parameter in (2) has two subcomponents in a given geographical area $i$ :

- the real-time effective reproduction factor $R_{t, i}$;

- estimated cease-fatality rate $(\mathrm{CRF}) C R F_{i}$.

The real-time effective reproduction factor $R_{t, i}$ is closely connected to the better-known basic reproduction number $R_{0}$. A rough estimation of dimensionless $R_{0}$ for a given geographical area $i$ is done taking into account transmissible power $\tau$, average infection-producing contacts per time $c$, and average infectious period $p$.

$$
R_{0}=\tau_{i} \times c_{i} \times p
$$

The last parameter $p$ can not be reduced (and usually it is not affected by geography and culture), but the first two can and they can depend on geography, culture, and other socio-economic characteristics of population at $i$. Massive and well-directed testing can, hopefully, find people who are infected in the early periods of infection, which could lead to reduced $c_{i}$ in a combination with an appropriate government response. The measures concerning self-isolation, social distancing, and disinfection can reduce $\tau_{i}$. Therefore, $R_{0}$ is not set in stone. It is an average, and can also vary from place to place, from time to time. It is a measure of disease potential.

On the other hand, the effective variant of $R_{0}$, denoted as $R_{t}$, should represent the actual transmission rate at a given time $t$, during an epidemic. Several method have been proposed to estimate $R_{0}$ using a field data [18-20], but they were usually difficult to generalize [21]. Cori et al. [21] have proposed a very simple technique to estimate $R_{t}$ using only incidence time series. For that they consider the number of new infections in $f_{t, i}$ observed at time $t$ for geographical area $i$, and the cumulative number of active cases at time step $t-1$ wighted with an infectivity function $w(s)$, expressed as $\sum_{s=1}^{t} i n f_{t-s, i} w(s)$. The weighted function $w(s)$ is basically a probability distribution of how infective person is at time step $s$. We have that

$$
R_{t, i}=\frac{i n f_{t, i}}{\sum_{s=1}^{t} i n f_{t-s, i} w(-s)} .
$$

Definition 2 For a geographical area $i$, CFR is defined as a ratio of deaths from a given disease and the total number of people which are diagnosed with the disease, in a certain period of time. 
Basically, the person who spreads the disease is unfortunately spreading lethality in an estimated percentage. For the particular epidemic, the best estimation of CFR is, obviously, done at the end of outbreak. In the mean time, a better way to evaluate this parameter is to do a forecast using well developed mathematical and data science techniques. Note that CFR is timedependent. Some of the reasons for this are:

- a misunderstanding of disease;

- seasonality;

- potential treatment can be found or improved during the epidemic.

Remark 2 SARS-CoV-2 has a rather long incubation period [22]. Therefore, person ceased at time step $t$ has been infected several days ago, i.e., at time step $t-s$, for some $s \leq t$. Therefore, a ratio of deceased at $t$ and confirmed cases at $t-s$ is a better suited way to estimate CFR, in order to derive a CFR function. Of course, in some cases, this could lead to very high lethality percentage estimation at the beginning of outbreak, which is why for the CFR the forecast should be considered.

Definition 3 Interpolation of CFR values at time steps $t \in T$ defines a CFR function.

Remark 3 There can be multiple CFR function for the same set of CFR values, when $t \in T$.

Remark 4 Another way to estimate CFR is to compute a ratio of deaths and closed cases. On the other hand, this method is affected by a government response. The obvious example is how this ratio looks like in Sweden and Russia during the SARS-CoV-2 pandemic 2020 [23].

Taking into account everything previously said, the Hazard parameter for a geographical area $i$ at time step $t$ could be evaluated as a product

$$
R_{t, i} \times C F R_{t, i} \times\left(i n f_{t, i}+\eta\right)
$$

where $C F R_{t, i}$ is a CFR value at time step $t$ and $\eta_{i} \in(0,1]$ is a corrective parameter, because we can not be certain that there are no infected persons at $i$.

The proposition to estimate $\eta_{i}$ is give as follows. In the beginning, $\eta$ can be some quite small random number, if there are no confirmed cases of disease. As the time passes by, it should slowly grow, even if there is still no confirmation of disease occurrence at $i$. It seems natural that growth rate of $\eta$ should follow the logistic function of epidemic growth. Thus, we should set $\eta=1$ when an epidemic reaches its peak, according to the forecasting model used, even if at $i$ there are still no confirmed cases. As a matter of fact, if there are confirmed cases, and the epidemic is at its peak, it is naturally to assume that there is at least one more person "out there" about which epidemiologists and management committee do not now about. In a way, this corrective parameter is a small precaution measure. 
Remark 5 The Hazard parameter is time-dependent.

Remark 6 The $\eta$ parameter is not dependent on $i \in I$, nor it is time-dependent.

Remark 7 In the rest of the paper, we will make a separate note when there is a need to distinguish larger geographical area, like a country, from a smaller one, like a municipality.

Remark 8 As theq Hazard looks like an absolute category parameter, it will be assumed that $\beta_{H}=1$.

\subsection{Exposure}

According to [6] the exposure to infectious agents related to human (and animal) is considered under the People at Risk (PaR) dimension. Any other hazard (like natural and man made hazards), which increases the susceptibility to infectious disease, is considered as the vulnerability. The higher the infectious hazard exposure the higher the risk, which is further aggravated by exposure to other hazards leading to further increase of the risk.

The PaR dimension represents, in a way, how much people are exposed to infectious agents. We should take into account that if there is no exposure, then no matter how sever the hazard event is, there is no risk. For a personto-person (P2P) borne diseases, a proxy indicators to estimate the exposure to pathogens is usually used [6].

There are factors that, obviously, emphasize P2P during the epidemic like a ratio of urban area percentage $u_{i}$ and population density $d_{i}$. These two parameters do not need to correlate, as household size can affect the $d_{i}$ in urban and non-urban areas. For example, high $d_{i}$ and low $u_{i}$ is obviously an increased risk situation. Therefore, it is natural to take them as a ratio $u d_{i}$. Also, there are factors that directly suppress the P2P dimension. Some of them are:

- average household size $\left(h h_{i}\right)$

- access to sanitation $\left(a 2 s_{i}\right.$, in percentages);

- access to clean water $\left(a 2 w_{i}\right.$, in percentages);

- access protective equipment per capita $\left(p e p c_{i}\right)$.

It is important to note that in the beginning phase of the epidemic outbreak population density reduces significantly in highly urban areas, as people are trying to get away from the hotspot. On the other hand, if the epidemic lasts too long, the parameters which correspond to mild suppression of the $\mathrm{P} 2 \mathrm{P}$ dimension could also decrease. Sometimes, even at the beginning of epidemic, shortages of protective equipment could happen [24].

Remark 9 These are not all parameters that should be taken into account. Considering which parameter to include in evaluation of Exposure should be based on some criteria. Here, two of those are identified: 
- availability of data;

- significance.

Therefore, the current parameter set is just given as an illustration.

\subsection{Vulnerability}

With vulnerability we describe how simply and how severely exposed people can be affected [6]. The impact of any epidemics in terms of numbers is morbidity (number of cases $c_{i}$ ) and mortality (number of deaths $m_{i}$ ) and its impact on socio-economic life. The vulnerability dimensions represent health vulnerability due to social, economic, illness, migratory and behavioral characteristics of the community.

Social parameters that can be considered are:

- demographic composition

- we can take the percentage of people older than 65 years $o_{i}$ for SARSCoV-2 epidemic (as they are the most vulnerable);

- the average age in population $\left(a_{i}\right)$;

- gender ratio, which for SARS-CoV-2 could be a male to female ratio $\left(m f r_{i}\right)$, as it seems that male population is in greater risk [25];

- the number of returnees from abroad $\left(\right.$ ret $\left._{i}\right)$, as it is proven that usually they were the ones who imported virus in the community [7].

Besides these parameters, it is natural to consider the following ones:

- the immigration process is happening alongside with the SARS-CoV-2 pandemic, so the number of immigrants $\left(i m g_{i}\right)$ should be taken into account, too;

- the offense per capita $i$ (of $\left.f_{i}\right)$, when assessing municipalities;

- the self-isolated persons per 1000 citizens $s i_{i}$;

- the number of penalties for breaking the self-isolation measurement per 1000 citizens $\left(\operatorname{sip}_{i}\right)$.

Remark 10 For SARS pandemic in 2003 the older generation had a better immune response, compared to the SARS-CoV-2 pandemic in 2020. While elderly people living in severely infected districts showed significantly lower levels of subjective wellbeing, these levels and those of the younger sample were found to remain within the normative range [26].

Economic parameters that can be taken into account are:

- aid per capita $\left(\right.$ aid $\left._{i}\right)$ - contribution as a percentage of gross national income;

- Gini coefficient $\left(\right.$ gini $\left._{i}\right)$;

- human development index $\left(h d i_{i}\right)$.

Remark 11 These parameters are not well suited for assessing the ERI on the municipality level. 
When we are talking about the illness, then the prevalence of identified risk categories is important. Particularly, for SARS-CoV-2 patients, the risk categories are constituted of people who have the following underlying medical conditions [27]:

- diabetes;

- pulmonary diseases such as asthma, chronic obstructive disease, tuberculosis;

- hypertension;

- liver disease;

- immunocompromised people;

- chronic kidney disease undergoing dialysis;

- severe obesity (body mass index (BMI) of 40 or higher).

One person can have multiple chronic underlaying conditions, i.e., we can have intersections in population. Therefore, it could be useful to have one parameter concerning illness $\left(i l l_{i}\right)$. Malnutrition $\left(m n_{i}\right)$ is also an important factor that can affect the reaction of government to epidemic, and it affects the population with and without health issues. Furthermore, the usual mortality should be included, although this is indirectly assumed in the HDI when we are computing ERI for the whole country (or state). In other words, on the level of municipality we can use the mortality parameter mort $t_{i}$. Other factors that could be considered are: air quality $\left(a q_{t, i}\right)$, air temperature $\left(a t_{t, i}\right)$, water quality $\left(w q_{i}\right)$, and smokers percentage $\left(s m k_{i}\right)$. The list is not exclusive.

Remark 12 It is expected that people living below the poverty line are more prone to develop chronic diseases earlier in their life.

Remark 13 It is reported in [28] the smokers were 1.4 times more likely $(\mathrm{RR}=1.4$, 95\% CI: $0.98-2.00)$ to have severe symptoms of COVID-19 and approximately 2.4 times more likely to be admitted to an ICU, need mechanical ventilation or die compared to non-smokers ( $\mathrm{RR}=2.4,95 \% \mathrm{CI}: 1.434 .04)$.

The following migration characteristics could be taken:

- the estimation of usual number of tourists from $j$ into $i$, which we could denote here as tour $_{i}^{j}$;

- the number of direct connections that $i$ has with the outside world (by road, railway, maritime, or air transport), which we could denote here as $\operatorname{conn}_{i}$

- internal net migration netmig $_{i}$.

In the early stage of epidemic, a government is inducing appropriate measures and policies to suppress the expansion of disease. For example, this means that passenger flights could be canceled [29] or whole cities could be put in a quarantine [30]. The more direct connections that $i$ has, the harder is to control all of them. On the other hand, in the later stages, government needs to assess where the measures can be weaken and how much, in order to give a fresh breath to the economy and social life of its citizens.

Remark 14 Similar observation holds for Vulnerability as in the case of Exposure parameter, concerning the Remark 9. 


\subsection{Coping Capacities}

Under coping capacities, according to [6], we understand necessary physical infrastructure, health system capacity, institutional and management capacity. To manage an epidemic in a better way, the government needs higher coping capacity in order to lower the ERI, according to (2). The factors that should be considered can be grouped in the following categories: governance and health infrastructure.

Governance parameters that can be considered are: Fragile State Index ( $f s i)$, Transparency and Corruption Index (tci), and government effectiveness. The last one is not quite easy to measure, furthermore it is hard to find a rough substitution.

Remark 15 These factors are only suited for the whole country or state level assessment.

According to INFORM [6], health infrastructure can decomposed into two categories: capacity and performance.

When it comes to capacities, the following parameters could be taken into account:

- the number of doctors per 1000 people $\left(d o c_{i}\right)$;

- the number of nurses per 1000 people $\left(\right.$ nur $\left._{i}\right)$;

- the number of hospital beds per 1000 people $b e d s_{i}$;

In some cases, more particular values could be important:

- the number of intensive case units per 1000 people $\left(i c u_{i}\right)$;

- the number of ventilators per 1000 people $\left(\right.$ vent $\left._{i}\right)$.

Remark 16 In technical report [6] the public health expenditure is taken into account, but in a way this is already included in the aid $_{i}$.

Considering the performance, a life expectancy (lif $e_{i}$ ), available number of vaccines $\left(\right.$ vacc $\left._{i}\right)$ and the number of pharmacies per 1000 citizens $\left(\right.$ pharm $\left._{i}\right)$ are factors that moderately increase the coping capacities. On the other hand, infant mortality $\left(\right.$ imort $\left._{i}\right)$ and maternal mortality $\left(\right.$ mmort $\left._{i}\right)$ are factors that obviously indicate lower performance of health care workers.

Remark 17 The parameter $v^{\prime} a c c_{i}$ does not need to be in a direct connection with a pathogen which caused the outbreak:

- if safe and well-tested vaccines for the particular disease are at disposal, than that is a direct method to suppress the epidemic;

- if there are no safe and well-tested vaccines for the particular disease, as in the case of SARS-CoV-2, then this parameter is concerned with other possible diseases and other epidemic outbreaks that can simultaneously happen (there can be two or more disease outbreaks in one period of time []).

Remark 18 Similar observation holds for Coping Capacities as in the case of Exposure parameter, concerning the Remark 9. 


\section{Epidemic Risk Index}

As we already know, the Hazard parameter, from now denoted as $h_{i}$ (for $i \in I)$, is computed as

$$
h_{t, i}=R_{t, i} \times C F R_{t, i} \times\left(i n f_{t, i}+\eta\right) .
$$

The Exposure parameter, from now denoted as $e_{i}$ (for $i \in I$ ), could be computed as

$$
e_{i}=\frac{u d_{i}}{h h_{i} \times a 2 s_{i} \times a 2 w_{i} \times p e p c_{i}}
$$

concerning the parameters discussed in Sect. 2. Similarly, the equation for $V$ ulnerability, form now denoted as $v_{i}$ (for $i \in I$ ), concerning the corresponding parameters, can be computed as the product of four variables

$$
v_{i}=\operatorname{soc}_{i} \times e c o_{i} \times i l l_{i} \times \operatorname{migr}_{i},
$$

where we have that, for example,

$$
\begin{aligned}
\text { soc }_{i} & =o_{i} \times a_{i} \times \text { mfr } \\
i & \times o f f_{i} \\
e c o_{i} & =\frac{\text { gini }_{i}}{\text { aid }_{i} \times h d i_{i}} \\
\text { ill }_{i} & =\text { ill }_{i} \times \text { mnut }_{i} \times \text { mort }_{i} \\
\text { migr }_{i} & =\text { conn }_{i} \times \sum_{j \in J}\left(h_{t, j} \times \text { tour }_{i}^{j}\right)
\end{aligned}
$$

One can see that some parameters discussed above are not included. Moreover, we have a cumulative sum for tourists weighted with the corresponding hazard parameter $h_{t, j}$. It is deliberately done, in order to illustrate the modularity and extensibility of proposed ERI. The Coping Capacities parameter, from now denoted as $c c_{t, i}$ (for $t \in T$ and $i \in I$ ), could be computed as

$$
c c_{t, i}=\operatorname{gov}_{i} \times \operatorname{cap}_{t, i} \times \operatorname{perf} f_{i}
$$

where we take that

$$
\begin{aligned}
\operatorname{gov}_{i} & =\frac{1}{f s i_{i} \times t c i_{i}} \\
\operatorname{cap}_{t, i} & =\operatorname{doc}_{t, i} \times \operatorname{nur}_{t, i} \times \text { beds }_{t, i} \times i c u_{t, i} \times \text { vent }_{t, i} \\
\operatorname{perf}_{i} & =\frac{\text { life }_{i} \times \operatorname{vacc}_{i} \times \text { pharm }_{i}}{\text { imort }_{i} \times \text { mmort }_{i}} .
\end{aligned}
$$

Remark 19 We have that $s o c_{i}$ is computed assuming that the lower $a_{i}$ is better for population, as it is suspected for SARS-CoV-2. If that is not the case, then $a_{i}$ and $o_{i}$ could, for example, switch places, or even be omitted.

Remark 20 For municipalities, $e \mathrm{co}_{i}$ and gov $_{i}$ should be omitted, i.e., replaced by 1 . 
Because the main four parameters can have quite different value ranges, it is natural to normalize them for all $i \in I$. For a parameter $x_{i}$ which takes non-negative values we can normalize it as

$$
X_{i}=\frac{x_{i}}{\max _{k \in I} x_{k}} .
$$

If a parameter $x_{i}$ can take negative values, i.e., values in range $\left[x_{\min }, x_{\max }\right]$, where $x_{\min } \leq 0$, then the corresponding normalized $X_{i}$ is obtained as

$$
X_{i}=\frac{x_{i}-x_{\min }}{\max _{k \in I} x_{k}-x_{\min }} .
$$

The normalized variants of parameters are denoted with capital letters. Therefore, the ERI for $i \in I$ can be computed as

$$
E R I_{t, i}=\varphi \times \frac{H_{t, i} \times E_{i}^{\beta_{E}} \times V_{i}^{\beta_{V}}}{C C_{t, i}^{\beta_{C C}}} \times \varepsilon
$$

where $H_{t, i}, E_{i}, V_{i}, C C_{t, i}$ correspond to normalized values of $h_{t, i}, e_{i}, v_{i}, c c_{t, i}$, respectively.

The fitting coefficients can be estimated by performing a non-linear leastsquare best-fit, minimizing the following error

$$
\sigma^{2}=\sum_{t \in T, i \in I}\left(C R F_{i} \times I N C_{t, i}-E R I_{t, i}\right)^{2}
$$

Here, $I N C_{t, i}$ represents the incidence, i.e., the probability of infection occurrence in a population within a specified period of time $t$. The first product $C R F_{t, i} \times I N C_{t, i}$ basically represents the current severity of epidemic at $i$.

The relative quadratic mean error is computed as

$$
\varepsilon^{2}=\frac{\sigma^{2}}{\sum_{t \in T, i \in I} C R F_{t, i}^{2} \times I N C_{t, i}^{2}} .
$$

Remark 21 For diseases in which CRF is extremely small, i.e., the CRF is almost zero, instead of $C R F_{i} \times I N C_{t, i}$ we could use some other measure, like the attack rate. The computation of relative quadratic mean error should be adjusted accordingly.

\section{Empirical study}

The computational experiments are conducted using data obtained from following two sources:

- official web-site of Government of Srpska Republic, regarding the SARSCoV-2 epidemic in Srpska [];

- Census 2013 data in Srpska Republic []. 
For computer programming and analysis tasks the Anaconda suite with Python 3.6 is used, installed on Windows 10 operating system. All figures in this work were produced using a Python package matplotlib [?].

In order to compute $E R I_{i}$ (for $i \in I$ ), the following parameters were considered: real-time effective reproductive number $R_{t, i}$, CFR $C F R_{t, i}$, new infections (confirmed cases) $i n f_{t, i}$, urban area percentage and population density ratio $u d_{i}$, average household size $h h_{i}$, percentage of people older than 65 years $o_{i}$, male to female ratio $m f r_{i}$, average age $a_{i}$, mortality mort $_{i}$, number of

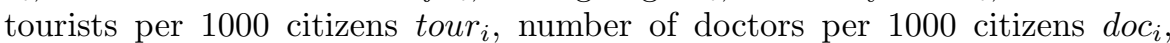
number of pharmacists per 1000 citizens pharm $_{i}$ (as data about pharmacies is not available), and infant mortality imort $_{i}$.

$R_{t}$ and $C F R_{t, i}$ are computed for the whole Srpska Republic, i.e., municipality level estimations are ignored. Following the findings in [] the basic reproductive number is set to $R_{0}=5,7$. $R_{t}$ is computed assuming that a Poisson model is appropriate, using the corresponding probabilistic mass function

$$
w(s)=\frac{R_{t-1, i}^{s} e^{-R_{t-1, i}}}{s !},
$$

where we have that a Poisson distribution with parameter $\lambda=R_{t-s, i}$, i.e., the number of transmissions in a average period from infection to confirmation corresponds to the latest $R_{t-1, i}$. CF $R_{t, i}$ at time $t$ is estimated by a ratio of deceased and confirmed cases at time $t-10$, as 10 is the average number of days from confirmation to the unwanted outcome. For time periods when $t-10<0$, the CFR value is extrapolated using the interpolation function. The corrective parameter $\eta_{i}$ is calculated as $\frac{t}{t_{\text {peak }}}$ when epidemic peak is predicted for the whole Srpska Republic.

It is assumed that every citizen has the access to sanitation, clean water, and basic protective equipment. Therefore, the exposure is computed as $\frac{u d_{i}}{h h_{i}}$.

The vulnerability is estimated using Eq. (??), where

$$
\begin{aligned}
\operatorname{soc}_{i} & =o_{i} \times a_{i} \times m f r_{i} \\
e c o_{i} & =1 \\
i l l_{i} & =1 \\
\operatorname{migr}_{i} & =\operatorname{conn}_{i} \times \sum_{j \in J}\left(h_{t, j} \times \operatorname{tour}_{i}^{j}\right) .
\end{aligned}
$$

For the coping capacities we have

$$
\begin{aligned}
\operatorname{gov}_{i} & =1 \\
\operatorname{cap}_{t, i} & =\text { doc }_{i} \\
\operatorname{perf} & =\frac{\text { life }_{i} \times \text { pharm }_{i}}{\text { imort }_{i}},
\end{aligned}
$$

where pharm $_{i}$ represents the number of pharmacists per 1000 citizens, as other data is not available. 


\section{Conclusion}

In this paper a novel way to estimate the ERI is presented. The idea how the ERI is developed leaves enough room for simple extension. For example, air quality and average temperature could easily be incorporated. The gravity model is used, as an specific upgrade of the INFORM methodology for assessing the risk.

This ERI is basically designed for diseases which are person-to-person airborne transmitted. Hopefully, this small research should be of immediate help to governments and health workers who are dealing with the current and future epidemics. Another possible usage of this formula could be in social sciences for analyzing the sociological effects of epidemics, government response, or population behavior, as the index is dimensionless.

\section{References}

1. Nigerian Federal Ministry of Health, National Policy on Integrated Disease Surveillance Response in Nigeria, 17, Federal Ministry of Health, Nigeria (2009)

2. Kapiriri, L., Ross, A. The Politics of Disease Epidemics: a Comparative Analysis of the SARS, Zika, and Ebola Outbreaks. Glob Soc Welf 7, 3345 (2020). https://doi.org/10.1007/s40609-018-0123-y

3. Fatiregun A, Isere E, Epidemic preparedness and management: A guide on Lassa fever outbreak preparedness plan, Niger Med J. 58(1):1-6. (2017) doi:10.4103/0300-1652.218414

4. World Health Organization, Managing epidemics: key facts about major deadly diseases, WHO, (2018)

5. Zhu M, Guo X, Lin Z The risk index for an SIR epidemic model and spatial spreading of the infectious disease, Math Biosci Eng. 14(5-6):1565-1583 (2017) doi:10.3934/mbe.2017081.

6. Doherty B, Marin-Ferrer M, Vernaccini L, INFORM Epidemic Risk Index, Technical report by the Joint Research Centre, the European Commissions science and knowledge service (2018)

7. Zlojutro, A., Rey, D. and Gardner, L. A decision-support framework to optimize border control for global outbreak mitigation. Sci Rep 9, 2216 (2019). https://doi.org/10.1038/s41598-019-38665-w

8. Haoxuan Sun, Yumou Qiu, Han Yan, Yaxuan Huang, Yuru Zhu, Song Xi Chen, Tracking and Predicting COVID-19 Epidemic in China Mainland, medRxiv (2020) doi:https://doi.org/10.1101/2020.02.17.20024257

9. World Health Organization, WHO Director-General's opening remarks at the media briefing on COVID-19 11 March 2020, (2020) url: https://www.who.int/dg/speeches/detail/who-director-general-s-opening-remarks-atthe-media-briefing-on-covid-19-11-march-2020

10. Jason Phua et al., Critical Care Bed Capacity in Asian Countries and Regions, Critical Care Medicine 20(30) (2020) doi:10.1097/CCM.0000000000004222

11. McCarthy N, The Countries With The Most Critical Care Beds Per Capita, Forbes, (2020) url:https://www.forbes.com/sites/niallmccarthy/2020/03/12/the-countries-withthe-most-critical-care-beds-per-capita-infographic/\#6d36296e7f86

12. Bittner J, Germany Has More Than Enough Ventilators. It Should Share Them, The New York Times, (2020) url:https://www.nytimes.com/2020/03/17/opinion/coronaviruseurope-germany.html

13. Poggioli S, For Help On Coronavirus, Italy Turns To China, Russia And Cuba, NPR, (2020) url: https://www.npr.org/sections/coronavirus-liveupdates/2020/03/25/821345465/for-help-on-coronavirus-italy-turns-to-china-russiaand-cuba 
14. Valero J, EU allocates Chinese aid to Italy to fight against the pandemic, EURACTIV.com, (2020) url: https://www.euractiv.com/section/coronavirus/news/eu-allocateschinese-aid-to-italy-to-fight-against-the-pandemic/

15. Augustin E, Cuba sends doctors worldwide in coronavirus fight, Al Jazeera (2020) url: https://www.aljazeera.com/news/2020/03/cuba-sends-doctors-worldwide-coronavirusfight-200330091411271.html

16. Carrre C, Mrzov M, Neary P.J, Gravity without Apology: The Science of Elasticities, Distance, and Trade, The Economic Journal, ueaa034, (2020) https://doi.org/10.1093/ej/ueaa034

17. Heesterbeek J, A brief history of $\mathrm{R} 0$ and a recipe for its calculation. Acta Biotheoretica 50(3):189-204.

18. Riley S, Fraser C, Donnelly CA, et al. Transmission dynamics of the etiological agent of SARS in Hong Kong: impact of public health interventions. Science. 300(5627):1961-1966 (2003)

19. Bettencourt LM, Ribeiro RM. Real time Bayesian estimation of the epidemic potential of emerging infectious diseases. PLoS One. 2008;3(5):e2185.

20. Cintron-Arias A, Castillo-Chavez C, Bettencourt LM, et al. The estimation of the effective reproductive number from disease outbreak data. Math Biosci Eng. 6(2):261282 (2009)

21. Cori A, Ferguson NM, Fraser C, Cauchemez S. A new framework and software to estimate time-varying reproduction numbers during epidemics. Am J Epidemiol. 2013 Nov 1;178(9):1505-12. doi: 10.1093/aje/kwt133. Epub 2013 Sep 15. PMID: 24043437; PMCID: PMC3816335.

22. LI Q, Guan X, Wu P, Wang X, et al. Early Transmission Dynamics in Wuhan, China, of Novel Coronavirus-Infected Pneumonia. N Engl J Med. 2020 Jan 29. doi: 10.1056/NEJMoa2001316.

23. WHO, Coronavirus disease 2019 (COVID-19) Situation Report 95, Data as received by WHO from national authorities by 10:00 CEST, 24 April 2020 url: https://www.who.int/docs/default-source/coronaviruse/situation-reports/20200424sitrep-95-covid-19.pdf?sfvrsn=e8065831_4

24. Connolly K, US hijacking mask shipments in rush for coronavirus protection, The Guardian (2020) url:https://www.theguardian.com/world/2020/apr/02/globalbattle-coronavirus-equipment-masks-tests

25. Jin J, Bai P, He W, Wu F, Liu X, Han D, Liu Sh, Yang J,Gender differences in patients with COVID-19: Focus on severity and mortality, medRxiv 2020.02.23.20026864; doi: https://doi.org/10.1101/2020.02.23.20026864

26. Lau AL1, Chi I, Cummins RA, Lee TM, Chou KL, Chung LW, The SARS (Severe Acute Respiratory Syndrome) pandemic in Hong Kong: effects on the subjective wellbeing of elderly and younger people, Aging Ment Health. 2008 Nov;12(6):746-60. doi: 10.1080/13607860802380607.

27. CDC, People Who Are at Higher Risk for Severe Illness, April 25th 2020, url: https://www.cdc.gov/coronavirus/2019-ncov/need-extra-precautions/people-at-higherrisk.html

28. Vardavas CI, Nikitara K. COVID-19 and smoking: A systematic review of the evidence. Tobacco Induced Diseases. 2020;18(March):20. doi:10.18332/tid/119324.

29. Jasper C, Philip S, Airlines Need Up to $\$ 200$ Billion to Survive Virus, IATA Says, Bloomberg, March 17, 2020, 2:45 PM GMT+1, url: https://www.bloomberg.com/news/articles/2020-03-17/airlines-need-up-to-200-bailoutto-survive-virus-iata-warns

30. Crossley G, Wuhan lockdown 'unprecedented', shows commitment to contain virus: WHO representative in China, REUTERS (2020) url: https://www.reuters.com/article/us-china-health-who-idUSKBN1ZM1G9

\section{A Appendix}

